\title{
LOWER BOUNDS FOR FUNDAMENTAL UNITS OF REAL QUADRATIC FIELDS
}

\author{
KOSHI TOMITA AND KOUJI YAMAMURO
}

\begin{abstract}
Let $d$ be a square-free positive integer and $l(d)$ be the period length of the simple continued fraction expansion of $\omega_{d}$, where $\omega_{d}$ is integral basis of $\mathbb{Z}[\sqrt{d}]$. Let $\varepsilon_{d}=\left(t_{d}+u_{d} \sqrt{d}\right) / 2(>1)$ be the fundamental unit of the real quadratic field $\mathbb{Q}(\sqrt{d})$. In this paper new lower bounds for $\varepsilon_{d}, t_{d}$, and $u_{d}$ are described in terms of $l(d)$. The lower bounds of $\varepsilon_{d}$ are sharper than the known bounds and those of $t_{d}$ and $u_{d}$ have been yet unknown. In order to show the strength of the method of the proof, some interesting examples of $d$ are given for which $\varepsilon_{d}$ and Yokoi's $d$-invariants are determined explicitly in relation to continued fractions of the form $\left[a_{0}, \overline{1, \ldots, 1, a_{l(d)}}\right]$.
\end{abstract}

\section{Introduction}

For a positive square-free integer $d$, let $D$ be the discriminant of the real quadratic field $\mathbb{Q}(\sqrt{d})$ and $l(d)$ be the period length in the simple continued fraction expansion of the algebraic integer $\omega_{d}=\left(\sigma_{d}-1+\sqrt{d}\right) / \sigma_{d}$, where $\sigma_{d}=1($ resp. 2) for $d \not \equiv 1(\bmod 4)(\operatorname{resp} . d \equiv 1(\bmod 4))$. It is well-known that the fundamental unit $\varepsilon_{d}=\left(t_{d}+u_{d} \sqrt{d}\right) / 2(>1)$ of $\mathbb{Q}(\sqrt{d})$ has lower bounds that increase with $l(d)$. For example, there are $((1+\sqrt{5}) / 2)^{l(d)}$ (see, for example [1, p. 240]) and $\sqrt{D}(3 / 2)^{l(d)-2}$ (see [2, p. 98]). For $d$ with sufficiently large $l(d), \varepsilon_{d}$ is much larger than these lower bounds. So we will caluculate a sharper lower bound in terms of $l(d)$ to study the sufficiently large $\varepsilon_{d}$ for $d$. Furthermore, we will calculate the lower bounds for $t_{d}$ and $u_{d}$ in terms of $l(d)$ that have been yet unknown. In order to study Yokoi's $d$-invariants, which are concerned with the class number one problem for real quadratic fields, we need to investigate $t_{d}$ and $u_{d}$. We have obtained the following theorem.

Received October 24, 2000.

2000 Mathematics Subject Classification: Primary 11R11; Secondary 11R27. 
THEOREM 1. Let $d$ be a positive square-free integer with $l(d) \geq 2$ and $D$ be the discriminant of $\mathbb{Q}(\sqrt{d})$. Then we have

$$
\varepsilon_{d}> \begin{cases}\frac{\sqrt{D}}{\sqrt{5}}\left(\frac{1+\sqrt{5}}{2}\right)^{l(d)-1} & \text { if } l(d) \text { is even, } \\ \frac{1}{\sqrt{5}}\left(\sqrt{D}-\frac{\sqrt{5}-1}{2}\right) \cdot\left(\frac{1+\sqrt{5}}{2}\right)^{l(d)} & \text { if } l(d) \text { is odd. }\end{cases}
$$

Moreover, for $t_{d}$ and $u_{d}$ in $\varepsilon_{d}=\left(t_{d}+u_{d} \sqrt{d}\right) / 2(>1)$, we have

$$
t_{d}> \begin{cases}\frac{\sqrt{D}}{\sqrt{5}}\left(\frac{1+\sqrt{5}}{2}\right)^{l(d)-1} & \text { if } l(d) \text { is even }, \\ \frac{1}{\sqrt{5}}(\sqrt{D}-\sqrt{5}+1) \cdot\left(\frac{1+\sqrt{5}}{2}\right)^{l(d)} & \text { if } l(d) \text { is odd }\end{cases}
$$

and

$$
u_{d}> \begin{cases}\frac{2}{\sigma_{d} \sqrt{5}}\left(\frac{1+\sqrt{5}}{2}\right)^{l(d)-1} & \text { if } l(d) \text { is even } \\ \frac{2}{\sigma_{d} \sqrt{5}}\left(\frac{1+\sqrt{5}}{2}\right)^{l(d)-2} & \text { if } l(d) \text { is odd } .\end{cases}
$$

In this paper, the simple continued fraction with period $l$ is generally denoted by $\left[a_{0}, \overline{a_{1}, \ldots, a_{l}}\right]$, and $[x]$ means the greatest integer less than or equal to $x$. Let $\left\{F_{i}\right\}$ be the Fibonacci numbers determined by $F_{0}=$ $0, F_{1}=1, F_{i+1}=F_{i}+F_{i-1}(i \geq 1)$. The Fibonacci numbers play an important role in showing Theorem 1 , because we use the inequality $\varepsilon_{d} \geq$ $\left(\left[\omega_{d}\right]+\omega_{d}\right) F_{l(d)}+F_{l(d)-1}\left(\operatorname{resp} .\left(\left[\omega_{d}\right]-1+\omega_{d}\right) F_{l(d)}+F_{l(d)-1}\right)$ in the case that $d \not \equiv 1(\bmod 4)(\operatorname{resp} . d \equiv 1(\bmod 4))$. We are interested in whether the equality is possible. We got an affirmative answer and we have discovered real quadratic fields with $m_{d} \neq 0$. Here $m_{d}$ is one of Yokoi's $d$-invariant, and it is defined by $m_{d}=\left[u_{d}^{2} / t_{d}\right]$. Another is defined by $n_{d}=\left[t_{d} / u_{d}^{2}\right]$. We know that there exist only finitely many $d$ satisfying both class number one and $n_{d} \neq 0$ (i.e. $m_{d}=0$ ) (see [7, p. 188]). So it is very important to investigate the case that $m_{d} \neq 0$ (i.e. $n_{d}=0$ ). Now these results are stated as follows: 
THEOREM 2. Let $d$ be a positive square-free integer and $l$ be a positive integer satisfying $l \geq 2$. Assume that

$$
d=\left(2 F_{l}+1\right)^{2}+8 F_{l-1}+4
$$

Then $d \equiv 1(\bmod 4), \omega_{d}=\left[F_{l}+1, \overline{1, \ldots, 1,2 F_{l}+1}\right]$, and $l(d)=l$ hold .

Moreover, we have

$$
\begin{gathered}
\varepsilon_{d}=\frac{1}{2}\left(2 F_{l}^{2}+F_{l}+2 F_{l-1}+F_{l} \sqrt{d}\right)\left(=\left(\left[\omega_{d}\right]-1+\omega_{d}\right) F_{l}+F_{l-1}\right), \\
\left\{\begin{array}{l}
t_{d}=2 F_{l}^{2}+F_{l}+2 F_{l-1}, \\
u_{d}=F_{l},
\end{array}\right.
\end{gathered}
$$

and

$$
n_{d}= \begin{cases}5 & \text { if } l=2 \\ 3 & \text { if } l=3 \\ 2 & \text { if } l \geq 5\end{cases}
$$

THEOREM 3. Let $d$ be a positive square-free integer and $l$ be a positive integer satisfying that $l \geq 2$ and $l \equiv 1,2$, or $4(\bmod 6)$. Assume that

$$
d=\left(\left(F_{l}+1\right) / 2\right)^{2}+F_{l-1}+1
$$

Then $d \not \equiv 1(\bmod 4), \omega_{d}=\left[\left(F_{l}+1\right) / 2, \overline{1, \ldots, 1, F_{l}+1}\right]$, and $l(d)=l$ hold.

Moreover, we have

$$
\begin{gathered}
\varepsilon_{d}=\frac{1}{2}\left(F_{l}^{2}+F_{l}+2 F_{l-1}+2 F_{l} \sqrt{d}\right)\left(=\left(\left[\omega_{d}\right]+\omega_{d}\right) F_{l}+F_{l-1}\right), \\
\left\{\begin{array}{l}
t_{d}=F_{l}^{2}+F_{l}+2 F_{l-1}, \\
u_{d}=2 F_{l},
\end{array}\right.
\end{gathered}
$$

and

$$
m_{d}= \begin{cases}1 & \text { if } l=2 \\ 2 & \text { if } l=4 \\ 3 & \text { if } l \geq 7\end{cases}
$$

Remark 1. Our aim is to show that, for any $l$, there exists $d$ satisfying $l(d)=l$ and $\omega_{d}=\left[a_{0}, \overline{1, \ldots, 1, a_{l(d)}}\right]$. The case that $l=6 n+1$ was treated in [4], but our proof is simpler than theirs. Their aim is to consider Eisenstein's problem. 


\section{Preliminary}

In order to prove our theorems, we need several lemmas.

LEMMA 1. For a square-free positive integer $d$, we suppose $\omega_{d}=$ $\left[a_{0}, \overline{a_{1}, \ldots, a_{l}}\right]$. Moreover let $q_{i}$ be the integers determined by $q_{0}=0, q_{1}=1$, $q_{i+1}=a_{i} q_{i}+q_{i-1}(i \geq 1)$. Then the fundamental unit $\varepsilon_{d}=\left(t_{d}+u_{d} \sqrt{d}\right) / 2$ $(>1)$ of $\mathbb{Q}(\sqrt{d})$ is given by the following formula:

If $d \not \equiv 1(\bmod 4)$, then

$$
\varepsilon_{d}=\left(a_{0}+\sqrt{d}\right) q_{l(d)}+q_{l(d)-1}, \quad\left\{\begin{aligned}
t_{d} & =2\left(a_{0} q_{l(d)}+q_{l(d)-1}\right), \\
u_{d} & =2 q_{l(d)} .
\end{aligned}\right.
$$

If $d \equiv 1(\bmod 4)$, then

$$
\varepsilon_{d}=\left(\frac{2 a_{0}-1+\sqrt{d}}{2}\right) q_{l(d)}+q_{l(d)-1}, \quad\left\{\begin{aligned}
t_{d} & =\left(2 a_{0}-1\right) q_{l(d)}+2 q_{l(d)-1}, \\
u_{d} & =q_{l(d)} .
\end{aligned}\right.
$$

Proof is omitted (see proof of Lemma 1 in [3]).

Lemma 2. For a positive square-free integer d, denote by $D$ the discriminant of $\mathbb{Q}(\sqrt{d})$. Then we have

$$
\varepsilon_{d}>(\sqrt{D}-1) F_{l(d)}+F_{l(d)-1} .
$$

Moreover, for $t_{d}$ and $u_{d}$ in $\varepsilon_{d}=\left(t_{d}+u_{d} \sqrt{d}\right) / 2(>1)$, we have

$$
t_{d}>(\sqrt{D}-2) F_{l(d)}+2 F_{l(d)-1} \quad \text { and } \quad u_{d} \geq\left(\frac{2}{\sigma_{d}}\right) F_{l(d)} .
$$

Proof. In the case that $d \not \equiv 1(\bmod 4)$, since $a_{0}>\sqrt{d}-1$ and $q_{i} \geq F_{i}$ for any integer $i(\geq 1)$, from Lemma 1 we get

$$
\begin{aligned}
\varepsilon_{d} & \geq\left(a_{0}+\sqrt{d}\right) F_{l(d)}+F_{l(d)-1} \\
& >(2 \sqrt{d}-1) F_{l(d)}+F_{l(d)-1} \\
& =(\sqrt{D}-1) F_{l(d)}+F_{l(d)-1} .
\end{aligned}
$$

For $t_{d}$ and $u_{d}$, in the case that $d \not \equiv 1(\bmod 4)$, we have

$$
t_{d}>2\left\{(\sqrt{d}-1) F_{l(d)}+F_{l(d)-1}\right\}=(\sqrt{D}-2) F_{l(d)}+2 F_{l(d)-1}
$$

and

$$
u_{d} \geq 2 F_{l(d)} .
$$

In the case that $d \equiv 1(\bmod 4)$, we get the results in the same way. 
We get the following lemma by straightforward calculations.

LEMMA 3. For $i \geq 1$,

$$
F_{i}> \begin{cases}\frac{1}{\sqrt{5}}\left(\frac{1+\sqrt{5}}{2}\right)^{i-1} & \text { if } i \text { is even } \\ \frac{1}{\sqrt{5}}\left(\frac{1+\sqrt{5}}{2}\right)^{i} & \text { if } i \text { is odd }\end{cases}
$$

\section{Proof of Theorem 1}

Proof of Theorem 1. We put $\alpha=(1+\sqrt{5}) / 2$. First we shall show the lower bound of $\varepsilon_{d}$. From Lemma 2, we know $\varepsilon_{d}>(\sqrt{D}-1) F_{l(d)}+F_{l(d)-1}$.

In the case that $l(d)(\geq 3)$ is odd, we have, from Lemma 3 ,

$$
\begin{aligned}
(\sqrt{D}-1) F_{l(d)}+F_{l(d)-1} & >(\sqrt{D}-1) \frac{\alpha^{l(d)}}{\sqrt{5}}+\frac{1}{\sqrt{5}} \alpha^{l(d)-2} \\
& =\frac{\alpha^{l(d)}}{\sqrt{5}}\left\{\sqrt{D}-\frac{\sqrt{5}-1}{2}\right\} .
\end{aligned}
$$

This proves the odd case.

In the case that $l(d)(\geq 2)$ is even, we have

$$
\begin{aligned}
(\sqrt{D}-1) F_{l(d)}+F_{l(d)-1} & >(\sqrt{D}-1) \frac{\alpha^{l(d)-1}}{\sqrt{5}}+\frac{\alpha^{l(d)-1}}{\sqrt{5}} \\
& =\frac{\sqrt{D}}{\sqrt{5}} \alpha^{l(d)-1} .
\end{aligned}
$$

Next we shall show the lower bounds of $t_{d}$ and $u_{d}$. From Lemma 2, we know

$$
t_{d}>(\sqrt{D}-2) F_{l(d)}+2 F_{l(d)-1} .
$$

In the case that $l(d)(\geq 3)$ is odd, we have

$$
\begin{aligned}
(\sqrt{D}-2) F_{l(d)}+2 F_{l(d)-1} & >(\sqrt{D}-2) \frac{\alpha^{l(d)}}{\sqrt{5}}+2 \frac{\alpha^{l(d)-2}}{\sqrt{5}} \\
& =\frac{\alpha^{l(d)}}{\sqrt{5}}(\sqrt{D}-\sqrt{5}+1) .
\end{aligned}
$$


In the case that $l(d)(\geq 2)$ is even, we have

$$
\begin{aligned}
(\sqrt{D}-2) F_{l(d)}+2 F_{l(d)-1} & >(\sqrt{D}-2) \frac{\alpha^{l(d)-1}}{\sqrt{5}}+2 \frac{\alpha^{l(d)-1}}{\sqrt{5}} \\
& =\frac{\sqrt{D}}{\sqrt{5}} \alpha^{l(d)-1} .
\end{aligned}
$$

From Lemma 2 and Lemma 3, we can get the lower bound of $u_{d}$ in a similar way as in the proof of $t_{d}$. Theorem has been completely proved.

From Theorem 1, we get the following corollary for the period $l(d)$ and Yokoi's $d$-invariant $m_{d}$ :

COROLlaRY. If there exist a positive integer $M$ and a positive squarefree integer $d$ such that $d>13$ and

$$
l(d) \geq \frac{\log (M+1)+\log (\sqrt{5 d})-\log 2}{\log \left(\frac{1+\sqrt{5}}{2}\right)}+1
$$

then $m_{d}>M$.

Proof. From the assumption, we have

$$
\frac{\sqrt{D}}{\sqrt{5}}\left(\frac{1+\sqrt{5}}{2}\right)^{l(d)-1} \geq(M+1) d
$$

Since it holds that

$$
\frac{1}{\sqrt{5}}\left(\sqrt{D}-\frac{\sqrt{5}-1}{2}\right) \cdot\left(\frac{1+\sqrt{5}}{2}\right)^{i}>\frac{\sqrt{D}}{\sqrt{5}}\left(\frac{1+\sqrt{5}}{2}\right)^{i-1}
$$

for any positive integer $i$, we get, from Theorem 1 ,

$$
\varepsilon_{d}>(M+1) d
$$

Moreover, $m_{d}=\left[\varepsilon_{d} / d\right]$ if $d>13$ from Theorem 1.1 in [8], therefore we have

$$
m_{d}>\frac{\varepsilon_{d}}{d}-1>M
$$


Remark 2. We describe the comparison between the lower bound in Theorem 1 and the two well-known lower bounds given in the introduction of this paper. From the proof of Corollary, our lower bound for $d$ with odd $l(d)$ is greater than $(\sqrt{D} / \sqrt{5}) \cdot((1+\sqrt{5}) / 2)^{l(d)-1}$. Moreover, if $D>13$, then we have the following:

$$
\frac{\sqrt{D}}{\sqrt{5}}\left(\frac{1+\sqrt{5}}{2}\right)^{l(d)-1}-\left(\frac{1+\sqrt{5}}{2}\right)^{l(d)}>0.22\left(\frac{1+\sqrt{5}}{2}\right)^{l(d)-1} .
$$

Furthermore, if $l(d) \geq 7$, then

$$
\frac{\sqrt{D}}{\sqrt{5}}\left(\frac{1+\sqrt{5}}{2}\right)^{l(d)-1}-\sqrt{D}\left(\frac{3}{2}\right)^{l(d)-2}>0.038 \sqrt{D}\left(\frac{1+\sqrt{5}}{2}\right)^{l(d)-2} .
$$

Hence, our lower bounds are sharper than theirs.

\section{Proof of Theorems 2 and 3}

Proof of Theorems 2 and 3. First we shall prove the first half of Theorem 3 . Suppose that $l \equiv 1,2$, or $4(\bmod 6)$. Since it holds that

$$
F_{l+6} \equiv F_{l} \quad(\bmod 4) \quad(l \geq 0),
$$

we have $d \not \equiv 1(\bmod 4)$. We put

$$
\omega_{R}=\left(F_{l}+1\right) / 2+\left[\left(F_{l}+1\right) / 2, \overline{\underbrace{\overline{1, \ldots, 1}}_{l-1}, F_{l}+1}\right] .
$$

Then we have

$$
\omega_{R}=F_{l}+1+\frac{1}{1}+\cdots+\frac{1}{1}+\frac{1}{\omega_{R}} .
$$

By a straightforward induction argument, we obtain that

$$
\omega_{R}=F_{l}+1+\frac{F_{l-1} \omega_{R}+F_{l-2}}{F_{l} \omega_{R}+F_{l-1}} .
$$

Here, using $F_{l}=F_{l-1}+F_{l-2}(l \geq 2)$, we have

$$
\omega_{R}^{2}-\left(F_{l}+1\right) \omega_{R}-\left(F_{l-1}+1\right)=0 .
$$

Since $\omega_{R}>0$, it holds that

$$
\omega_{R}=\frac{F_{l}+1}{2}+\sqrt{d}
$$


Hence, we obtain that $\omega_{d}=\left[\left(F_{l}+1\right) / 2, \overline{1, \ldots, 1, F_{l}+1}\right]$ and $l(d)=l$, and we can determine $\varepsilon_{d}, t_{d}$ and $u_{d}$ from Lemma 1 . In the case of Theorem 2 , we put

$$
\omega_{R}=F_{l}+\left[F_{l}+1, \overline{\underbrace{1, \ldots, 1}_{l-1}, 2 F_{l}+1}\right] .
$$

Since we have $\omega_{R}=F_{l}+\omega_{d}$ in the same way, we can determine $\varepsilon_{d}, t_{d}$ and $u_{d}$.

Next we shall show the remaining part of Theorem 3 . Since $1 / F_{l}$ is monotone decreasing in $l$, it holds that

$$
3.4<4\left(1+\frac{3}{F_{l}}\right)^{-1} \leq 4\left(1+\frac{1}{F_{l}}+\frac{2 F_{l-1}}{F_{l}^{2}}\right)^{-1}<4
$$

for $l \geq 7$. Hence, we have $m_{d}=3$ for $l \geq 7$ from definition of $m_{d}$. And we can get $m_{d}$ in the case that $l=2$ or 4 from straightforward calculations.

Lastly we shall show the remaining part of Theorem 2 . We have $n_{d}=2$ for $l \geq 5$ from the definition of $n_{d}$, because

$$
2 \leq n_{d} \leq 2+\frac{1}{F_{l}}+\frac{2 F_{l-1}}{F_{l}^{2}}<2+\frac{3}{F_{l}}<3
$$

for $l \geq 5$. And we can get $n_{d}$ in the case that $l=2$ or 3 from straightforward calculations. Theorems have been proved.

Table: Square-free positive integers $d$ with $2<l(d) \leq 15$ represented by the Fibonacci numbers:

\begin{tabular}{|r|c|r|r|r|l|}
\hline$d$ & $\begin{array}{c}l(d) \\
(\bmod 6)\end{array}$ & $l(d)$ & $h_{d}$ & $F_{l(d)}$ & $\omega_{d}$ \\
\hline 3 & 2 & 2 & 1 & 1 & {$[1, \overline{1,2}]$} \\
21 & 2 & 2 & 1 & 1 & {$[2, \overline{1,3}]$} \\
37 & 3 & 3 & 1 & 2 & {$[3, \overline{1,1,5}]$} \\
7 & 4 & 4 & 1 & 3 & {$[2, \overline{1,1,1,4}]$} \\
69 & 4 & 4 & 1 & 3 & {$[4, \overline{1,1,1,7}]$} \\
149 & 5 & 5 & 1 & 5 & {$[6, \overline{1,1,1,1,11}]$} \\
58 & 1 & 7 & 2 & 13 & {$[7, \overline{1,1,1,1,1,1,14}]$} \\
797 & 1 & 7 & 1 & 13 & {$[14, \overline{1,1,1,1,1,1,27}]$} \\
4933 & 3 & 9 & 3 & 34 & {$[35, \overline{1,1,1,1,1,1,1,1,69}]$} \\
32485 & 5 & 11 & 8 & 89 & {$[90, \overline{1,1,1,1,1,1,1,1,1,1,179}]$} \\
84237 & 0 & 12 & 6 & 144 & {$[145, \overline{1,1,1,1,1,1,1,1,1,1,1,289}]$} \\
\hline
\end{tabular}


Table: Square-free positive integers $d$ with $2<l(d) \leq 15$ represented by the Fibonacci numbers:

\begin{tabular}{|r|c|r|r|r|l|}
\hline$d$ & $\begin{array}{c}l(d) \\
(\bmod 6)\end{array}$ & $l(d)$ & $h_{d}$ & $F_{l(d)}$ & $\omega_{d}$ \\
\hline 13834 & 1 & 13 & 22 & 233 & {$[117, \overline{1,1,1,1,1,1,1,1,1,1,1,1,234}]$} \\
219245 & 1 & 13 & 12 & 233 & {$[234, \overline{1,1,1,1,1,1,1,1,1,1,1,1,467}]$} \\
1493861 & 3 & 15 & 20 & 610 & {$[611, \overline{1,1,1,1,1,1,1,1,1,1,1,1,1,1,1221]}$} \\
\hline
\end{tabular}

Finally, we have the above table for $d$ concerning Theorem 2 and Theorem 3. Here $h_{d}$ is the class number of $\mathbb{Q}(\sqrt{d})$.

\section{REFERENCES}

[1] R. A. Mollin, Quadratics, CRC Press, Boca Rato, FL., 1996.

[2] R. Sasaki, A characterization of certain real quadratic fields, Proc. Japan Acad., 62, Ser. A (1986), no. 3, 97-100.

[3] K. Tomita, Explicit representation of fundamental units of some quadratic fields, Proc. Japan Acad., 71, Ser. A (1995), no. 2, 41-43.

[4] K. S. Williams and N. Buck, Comparison of the lengths of the continued fractions of $\sqrt{D}$ and $\frac{1}{2}(1+\sqrt{D})$, Proc. Amer. Math. Soc., 120 (1994), no. 4, 995-1002.

[5] H. Yokoi, The fundamental unit and class number one problem of real quadratic fields with prime discriminant, Nagoya Math. J., 120 (1990), 51-59.

[6] H. Yokoi, a note on class number one problem for real quadratic fields, Proc. Japan Acad., 69, Ser. A (1993), 22-26.

[7] H. Yokoi, The fundamental unit and bounds for class numbers of real quadratic fields, Nagoya Math. J., 124 (1991), 181-197.

[8] H. Yokoi, New invariants and class number problem in real quadratic fields, Nagoya Math. J., 132 (1993), 175-197.

Koshi Tomita

Department of Mathematics

Meijo University

Shiogamaguchi 1-501, Tenpaku-ku, Nagoya, 468-8502

Japan

tomita@ccmfs.meijo-u.ac.jp

Kouji Yamamuro

Department of Liberal Arts

Aichi Konan College

Omatsubara 172, Takaya-cho, Konan-shi, Aichi, 483-8086

Japan

yamamuro@math.nagoya-u.ac.jp 